\title{
X-ray emission of brown dwarfs: towards constraining the dependence on age, luminosity, and temperature
}

\author{
B. Stelzer ${ }^{1,2}$, G. Micela ${ }^{2}$, E. Flaccomio ${ }^{2}$, R. Neuhäuser ${ }^{3}$, and R. Jayawardhana ${ }^{4}$
}

\author{
1 Dipartimento di Scienze Fisiche ed Astronomiche, Università di Palermo, Piazza del Parlamento 1, 90134 Palermo, Italy \\ e-mail: stelzer@astropa.unipa.it \\ 2 INAF - Osservatorio Astronomico di Palermo, Piazza del Parlamento 1, 90134 Palermo, Italy \\ 3 Astrophysikalisches Institut und Universitäts-Sternwarte Jena, Schillergässchen 2-3, 07745 Jena, Germany \\ ${ }^{4}$ Department of Astronomy \& Astrophysics, University of Toronto, Toronto, ON M 5S 2H8, Canada
}

Received 21 June 2005 / Accepted 5 October 2005

\section{ABSTRACT}

\begin{abstract}
Aims. We observed brown dwarfs in different evolutionary stages with the Chandra X-ray Observatory with the aim to disentangle the influence of different stellar parameters on the X-ray emission of substellar objects. The ages of our three targets (HR 7329 B, G1569 Bab, and HD 130948 BC) are constrained by them being companions to main-sequence stars of known age. With both known age and effective temperature or bolometric luminosity, the mass can be derived from evolutionary models.

Methods. Combining the new observations with previous studies presented in the literature yields a brown dwarf sample that covers the age range from $\sim 1 \mathrm{Myr}$ to $\sim 1 \mathrm{Gyr}$. Since the atmospheric temperature of brown dwarfs is approximately constant at young ages, a sample with a large age spread is essential for investigating the possible influence of effective temperature on X-ray activity.

Results. Two out of three brown dwarfs are detected with Chandra, with variable lightcurves and comparatively soft spectra. Combining our results with published data allows us to consider a subsample of high-mass brown dwarfs (with $0.05-0.07 M_{\odot}$ ), thus eliminating mass from the list of free parameters. We find evidence that X-ray luminosity declines with decreasing bolometric luminosity steeper than expected from the canonical relation for late-type stars $\left(L_{\mathrm{x}} / L_{\mathrm{bol}}=10^{-3 \ldots-5}\right)$. Effective temperature is identified as a likely parameter responsible for the additional decline of X-ray activity in the more evolved (and therefore cooler) brown dwarfs of the "high-mass" sample. In another subsample of brown dwarfs characterized by similar effective temperature, the X-ray luminosity scales with the bolometric luminosity without indications for a deviation from the canonical range of $10^{-3 \ldots-5}$ observed for late-type stars.

Conclusions. Our findings support the idea that effective temperature plays a critical role for the X-ray activity in brown dwarfs. This underlines an earlier suggestion based on observations of chromospheric $\mathrm{H} \alpha$ emission in ultracool dwarfs that the low ionization fraction in the cool brown dwarf atmospheres may suppress magnetic activity.
\end{abstract}

Key words. X-rays: stars - stars: low-mass, brown dwarfs - stars: coronae - stars: activity - stars: general

\section{Introduction}

In the last few years a large number of very low-mass (VLM) dwarf stars and brown dwarfs (BD) have been discovered in the solar neighborhood by sky surveys such as 2 MASS, DENIS, or SDSS. Spectroscopic follow-up of these objects has enabled the first systematic investigation of chromospheric $\mathrm{H} \alpha$ activity in stars of the very smallest masses and even BDs (Gizis et al. 2000; Mohanty \& Basri 2003). One of the most important results of these studies is that $\mathrm{H} \alpha$ emission seems to reach a maximum at spectral type M 7, and fade off for the latest M dwarfs suggesting a change in the efficiency of the mechanism that drives magnetic activity.

$\mathrm{X}$-ray emission is a complementary activity indicator for late-type stars, probing the interplay between magnetic field and matter in the outermost and hottest part of the atmosphere, the corona. Hünsch et al. (1999) have presented a study of X-ray emission on field M dwarfs based on the ROSAT All-Sky Survey providing detections for spectral type earlier than M 7 . But beyond spectral type M 6 the X-ray regime is widely unexplored. Only a handful of late $\mathrm{M}$ dwarfs have been detected with the EXOSAT and/or ROSAT missions. Except LHS 2065, for which Schmitt \& Liefke (2002) detected quiescent emission with the ROSAT HRI, all these objects were only detected during a flare (Giampapa et al. 1996; Fleming et al. 2000). The coolest field object detected in X-rays so far is LP 944-20, an intermediate age M9.5 BD ( $t \sim 500 \mathrm{Myr}$; Tinney 1998). While ROSAT had provided a comparatively high upper limit (Neuhäuser et al. 1999), Chandra has seen LP944-20 during a flare (Rutledge et al. 2000), and XMM-Newton has provided the lowest upper limit for the quiescent flux of any field dwarf so far: $L_{\mathrm{x}}<3.1 \times 10^{23} \mathrm{erg} / \mathrm{s}$ (Martín \& Bouy 2002). Deep 
X-ray studies have been attempted for only few L dwarfs: Denis J1228-1547 (an L5 binary) remained undetected in an XMM-Newton observation, which was effectively quite shallow due to high background resulting from solar flares (Stelzer $\&$ Neuhäuser 2002). Berger et al. (2005) presented Chandra observations of two nearby L dwarfs, an upper limit of $L_{\mathrm{x}}<$ $6.6 \times 10^{24} \mathrm{erg} / \mathrm{s}$ for 2MASS J1507-1627 (SpT L3.5) and of $L_{\mathrm{x}}<9.3 \times 10^{24} \mathrm{erg} / \mathrm{s}$ for 2MASS J0036+1821 (SpT L5). The latter one was observed simultaneously in various wavebands including radio, optical and X-rays. Finally, the T dwarf binary $\epsilon$ Ind Bab was observed but not detected with Chandra, yielding an upper limit of $L_{\mathrm{x}}<3.2 \times 10^{23} \mathrm{erg} / \mathrm{s}$ (Audard et al. 2005).

While the observational material on X-ray activity of ultra-cool field dwarf stars and BDs is scarse, X-ray emission has lately been revealed from a substantial number of 1-5 Myr-old VLM pre-MS stars and BDs in various star forming regions (e.g. Neuhäuser \& Comerón 1998; Preibisch \& Zinnecker 2002; Mokler \& Stelzer 2002; Feigelson et al. 2002; Getman et al. 2002; Stelzer et al. 2004; Preibisch et al. 2005a), for one BD in the $\sim 10$ Myr-old TW Hya association (Tsuboi et al. 2003), one $~ 5 \mathrm{Myr}$ old member of Upper Sco (Bouy 2004), and one BD in the Pleiades (Briggs \& Pye 2004).

BDs are not able to fuse normal hydrogen and subsequently must cool down and become fainter as they age. Therefore, their detection at young ages but non-detection in more evolved stages suggests a connection between activity and effective temperature or bolometric luminosity. Based on the absence or low level of $\mathrm{H} \alpha$ emission seen in most field L dwarfs Mohanty et al. (2002) have argued that the chromospheric emission may shut off below a critical temperature because the atmosphere becomes too neutral to provide substantial coupling between matter and magnetic field. But due to a lack of systematic observations this hypothesis has not been tested so far. On the other hand, the X-ray luminosity of late-type stars in the solar neighborhood with spectral type earlier than $\sim \mathrm{M} 6$ is seen to decrease along the spectral type sequence, while $L_{\mathrm{x}} / L_{\mathrm{bol}}$ remains approximately constant at $\sim-4$ with a spread of $\sim 2$ dex for each spectral type subclass; see e.g. Fig. 4 in Stelzer (2004). If the $L_{\mathrm{x}}-L_{\mathrm{bol}}$-relation of late-type stars proceeds into the cool end of the MS and beyond the substellar limit the non-detection of most VLM field stars and BDs with the previously available low-sensitivity X-ray instruments is no surprise. However, as we will show in this paper Chandra is capable of providing meaningful constraints for the X-ray emission of these faint objects.

\section{Sample}

We have embarked on a systematic Chandra study of VLM stars and BDs with the aim to constrain the influence of stellar parameters on their X-ray emission. One of the prerequisites for our project is to know the age and effective temperature and/or bolometric luminosity of the BDs. The mass can then be deduced from evolutionary models. Both $T_{\text {eff }}$ and $L_{\text {bol }}$ decrease rapidly with age. Therefore, is it important to examine evolved BDs, and compare their X-ray properties to those of young BDs in star forming regions. To this end, we have compiled from the literature X-ray luminosities and

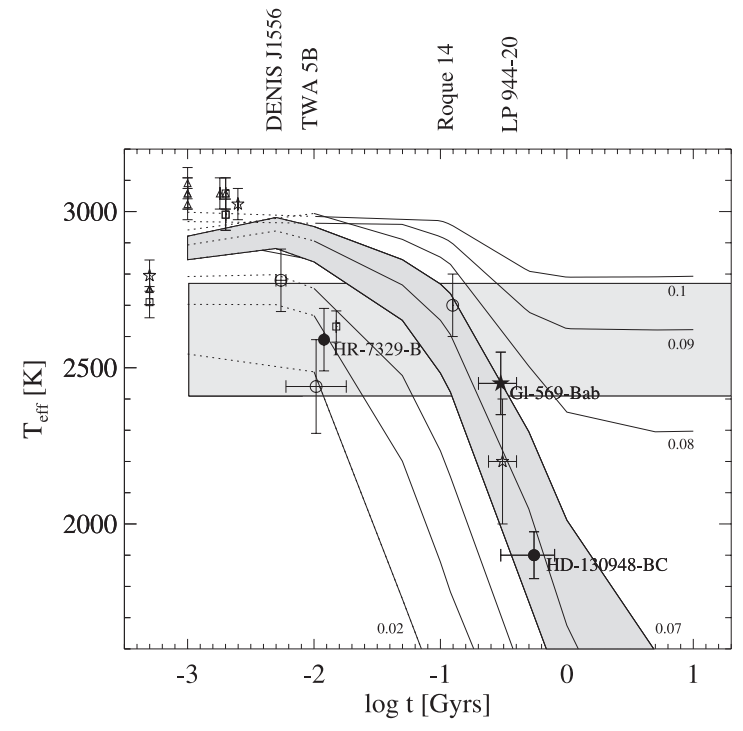

Fig. 1. BDs and BD candidates with known age on the model tracks from Baraffe et al. (1998) and Chabrier et al. (2000) in steps of $0.01 M_{\odot}$. The $0.08 M_{\odot}$ track marks the separation from stars to BDs. Only objects that were detected with XMM-Newton or Chandra are shown. These include: squares - BDs in IC 348; triangles - BDs in Cha I; open circles - further BDs compiled from the literature, see labels on top of the figure; filled symbols - BDs observed in the Chandra program described in this paper. Objects on which an X-ray flare was detected are marked by a star symbol. The grey-shades indicate distinct mass and temperature ranges we study in this paper.

stellar parameters for BDs in various evolutionary stages, and combine these data with our new Chandra observations.

Figure 1 shows the socalled cooling curves (effective temperature versus age for objects of given mass) according to the model calculations by Baraffe et al. (1998) and Chabrier et al. (2000). The data points represented by open plotting symbols refer to BDs from XMM-Newton or Chandra observations compiled from the literature. For clarity we show only BDs that were detected with XMM-Newton or Chandra. Non-detections are not displayed in Fig. 1, but will be included in the discussion later on. Filled plotting symbols denote targets from our new Chandra observations. As summarized in Sect. 1, previous X-ray satellites did not yield meaningful constraints on BD $\mathrm{X}$-ray emission, with the exception of some very young BDs, and their results will not be repeated here.

As is clear from Fig. 1 the cooling curves are poorly populated with X-ray data, especially at evolved ages. Several of the X-ray observed BDs mentioned in Sect. 1 do not show up in Fig. 1 because their age is not known. This is because the age determination in the coolest part of the HR diagram is subject to considerable uncertainty for two reasons: (i) For BDs there is always a degeneracy in the mass-age plot because they do not reach the MS. (ii) Theoretical evolutionary models for VLM stars and BDs start with assumed initial conditions, so that the results are quite uncertain for the first 10 to $100 \mathrm{Myr}$, and they have not been tested nor calibrated for VLM objects.

To circumvent the problem of the age determination for the BDs we have selected our Chandra sample among substellar companions to MS stars. Ages of MS stars can be 
Table 1. Sample of BD companions to MS stars observed with Chandra ACIS-S. The left hand side of the table represents a summary of stellar parameters. The distances are from Hipparcos, references for the other parameters are given in brackets. On the right hand side we list X-ray properties derived from the Chandra data: X-ray luminosity, ratio of X-ray to bolometric luminosity attributing all X-ray emission to either of the components for unresolved binaries, and median photon energy. The labels " $\mathrm{A}$ ", "Q", and "F" denote average, quiescent, and flare luminosities.

\begin{tabular}{|c|c|c|c|c|c|c|c|c|c|c|c|c|c|}
\hline Name & $\begin{array}{c}\text { Sep } \\
{\left[{ }^{\prime \prime}\right]} \\
\end{array}$ & $\begin{array}{l}\text { PA } \\
{\left[{ }^{\circ}\right]} \\
\end{array}$ & $\begin{array}{l}\text { Dist } \\
{[\mathrm{pc}]}\end{array}$ & SpT & $\begin{array}{c}M \\
{\left[M_{\odot}\right]}\end{array}$ & $\begin{array}{c}T_{\text {eff }} \\
{[\mathrm{K}]}\end{array}$ & $\begin{array}{l}\text { Age } \\
{[\mathrm{Gyr}]}\end{array}$ & $\log \frac{L_{\mathrm{bol}}}{L_{\odot}}$ & Ref. & & $\begin{array}{c}-8 \mathrm{keV}] \\
\log L_{\mathrm{x}} \\
{[\mathrm{erg} / \mathrm{s}]}\end{array}$ & $\log \frac{L_{\mathrm{X}}}{L_{\mathrm{bol}}}$ & $\left\langle E_{\mathrm{ph}}\right\rangle$ \\
\hline HR $7329 \mathrm{~B}$ & 4.097 & 166.9 & 47.7 & M 7.5 & $0.020-0.045$ & 2600 & 0.012 & -2.59 & $(1,2)$ & A & 27.2 & -3.8 & 0.87 \\
\hline \multirow[t]{3}{*}{ Gl $569 \mathrm{Bab}$} & 4.89 & 30.0 & 9.8 & & & & $0.2-0.4$ & & (3) & A & 26.8 & & 0.85 \\
\hline & & & & & & & & & & Q & 25.8 & & 0.67 \\
\hline & & & & & & & & & & $\mathrm{F}$ & 27.5 & & 0.87 \\
\hline $\mathrm{Ba}$ & & & & M 8.5 & $0.055-0.087$ & 2450 & & -3.35 & (4) & $\mathrm{A}$ & & -3.2 & \\
\hline $\mathrm{Bb}$ & & & & M9 & $0.034-0.070$ & 2450 & & -3.58 & (4) & A & & -3.4 & \\
\hline HD 130948 BC & 2.64 & 104.5 & 17.9 & & & & $0.3-0.8$ & & (5) & A & $<25.6$ & & - \\
\hline $\mathrm{B}^{*}$ & & & & L4 & $0.040-0.065$ & 1900 & & -3.81 & $(5,6)$ & A & & $<-4.2$ & \\
\hline $\mathrm{C}^{*}$ & & & & L4 & $0.040-0.065$ & 1900 & & -3.93 & $(5,6)$ & A & & $<-4.1$ & \\
\hline
\end{tabular}

References: (1) Guenther et al. (2001), (2) Lowrance et al. (2000), (3) Lane et al. (2001), (4) Zapatero Osorio et al. (2004).

(5) Potter et al. (2002), (6) Goto et al. (2002);

* Bolometric luminosity estimated from the $K$ band magnitude and the bolometric correction for the $K$ band given by Leggett et al. (2001).

estimated by a number of methods, e.g. space motion, metallicity, rotation, and activity (e.g. Lachaume et al. 1999), and companions are expected to be coeval. Within our program Chandra has observed three BD companions that span a significant range of ages $(\sim 12-550 \mathrm{Myr}), T_{\text {eff }}(2600-1900 \mathrm{~K})$, $\log L_{\text {bol }}(29.7-31.0 \mathrm{erg} / \mathrm{s})$, and masses $\left(0.03-0.07 M_{\odot}\right)$; filled circles in Fig. 1. The youngest of them, HR 7329 B, connects to the sequence of BDs in young stellar associations and star forming regions. The other two targets (HD $130948 \mathrm{BC}$ and Gl $569 \mathrm{Bab}$ ) have similar age, but $T_{\text {eff }}$ differs by $\sim 500 \mathrm{~K}$ (corresponding to $\sim 5$ spectral subclasses) and $L_{\text {bol }}$ differs by a factor of $\sim 20$, as a result of the strong dependence of these parameters on mass for evolved BDs. The Chandra observation of G1 569 Bab has already been discussed in detail by Stelzer (2004). We include here the previous results, in addition to some further X-ray parameters not given in Stelzer (2004). The stellar parameters and X-ray properties of all BD targets are summarized in Table 1.

We complement this data set by BDs presented in the XMM-Newton and Chandra literature, for which the age is known. The X-ray literature comprises a number of nearby low-mass star forming regions. We include in our study only IC 348 and Cha I. The stellar populations of these two regions were shown to be compatible with the Baraffe et al. (1998) and Chabrier et al. (2000) models. For somewhat younger regions, such as $\rho$ Oph and ONC, many objects lie above the youngest isochrone in these models (1 Myr), such that we can not place them in Fig. 1. Furthermore, the stellar parameters for BDs in IC 348 and Cha I presented in the literature, (spectral type, effective temperature and bolometric luminosity) were derived in the same manner for both regions, thus yielding a homogeneous data set. Specifically, the spectral types were determined by comparing the optical low-resolution spectra to those of standard dwarfs and giants. And the temperature scale used for the conversion of spectral types to $T_{\text {eff }}$ was devised by Luhman et al. (2003) for use with the Baraffe et al. (1998) and Chabrier et al. (2000) models.

Our X-ray selected sample of IC 348 is from Preibisch \& Zinnecker (2002) and that of Cha I from Stelzer et al. (2004). We consider objects with spectral type M7 and later as bonafide BDs, and those with spectral type M5 to M7 as BD candidates. For simplicity we refer to both of them as "BDs" throughout this paper. For IC 348 the stellar parameters are taken from Luhman (1999) and Luhman et al. (2003). In the latter paper Luhman established that four of the BD candidates in IC 348 identified by Najita et al. (2000) and included in the X-ray study of Preibisch \& Zinnecker (2002) are possibly field stars (N021-05, N024-02, N071-02, N075-01). Furthermore, N013-05 is classified as an M3 star by Luhman et al. (2003), and therefore not a BD. We exclude these five objects from our study. For Cha I we use the stellar parameters from the compilation by Luhman (2004). In order to put the young BDs in Fig. 1 we estimated ages on an individual basis from the position of each object on the Baraffe et al. (1998) and Chabrier et al. (2000) isochrones in the HR diagram. BDs above the youngest isochrone are arbitrarily placed at an age of $0.5 \mathrm{Myr}$ in Fig. 1, because for such young objects the evolutionary calculations are sensitive to the (unknown) initial conditions and therefore unconstrained (Baraffe et al. 2002). Actually, the models are uncertain for ages up to a few Myr. To indicate that the tracks for young ages must be used with caution, they are drawn with dotted lines in Fig. 1. We stress that the results derived in our study do not depend sensitively on these uncertainties; see discussion in Sect. 5.1.

Besides these young BDs in star forming regions XMM-Newton or Chandra data have been published for the 
following BDs with reliable age estimates from their membership in associations or clusters: DENIS J1556 in Upper Sco (5-6 Myr), TWA 5B and 2M 1207-39 in TW Hya ( 10 Myr), and five BDs in the Pleiades ( $\sim 125 \mathrm{Myr})$; see Sect. 1 for references to the X-ray data. In the solar neighborhood the number of BDs and VLM stars with known age is very limited, due to the problems outlined above. X-ray data have been presented for LP 944-20, APMPM J2354, and $\epsilon$ Ind Bab. For LP 944-20 we adopt an age of $320 \pm 80 \mathrm{Myr}$, derived from its association with the Castor moving group (Ribas 2003). Note that this is lower than the previous age estimate based on its lithium equivalent width (Tinney 1998). APMPM J2354 is a proper motion companion to a binary composed of a M 4 dwarf star and a white dwarf (Scholz et al. 2004). The age of the system is $\sim 1.8 \mathrm{Gyr}$ determined from the cooling history of the white dwarf (Silvestri et al. 2001). APMPM J2354 is the only star in our sample, and it is included in this study in order to examine the $\mathrm{X}$-ray properties across the substellar boundary. The $\mathrm{T}$ dwarf binary $\epsilon$ Ind Bab has an age of $0.2-2$ Gyr estimated from the rotation properties of the primary $\epsilon$ Ind A (Lachaume et al. 1999).

For the objects introduced in the previous paragraph the bolometric luminosity and the spectral type are compiled from the literature. We have derived their effective temperature from the spectral type using the scale given by Mohanty \& Basri (2003). We caution that the temperatures of the younger BDs $(\leq 100 \mathrm{Myr})$ with lower gravity may be somewhat higher than inferred with this scale which was devised for dwarfs. But within the estimated uncertainties this does not affect our study. The values for $T_{\text {eff }}$ obtained this way are compatible with the temperatures predicted by the Baraffe et al. (1998) and Chabrier et al. (2000) models, except for LP 944-20, where there is a discrepancy of $400 \mathrm{~K}$; see also discussion in (Ribas 2003). For this BD we assume a temperature of $2200 \pm 200 \mathrm{~K}$, intermediate between the empirical scale and the evolutionary calculations.

\section{Observations and data analysis}

The observing logs for our Chandra observations are summarized in Table 2. All three targets were observed with the Advanced CCD Imaging Spectrometer (ACIS), using the S3 chip in imaging mode. The data reduction for HR 7329 and HD 130948 was carried out using the CIAO software package $^{1}$ version 3.2 in combination with the calibration database (CALDB) version 3.0.0, in an analogous way to the analysis of the data for Gl 569 which was already presented by Stelzer (2004).

We started the analysis with the level 1 events file provided by the pipeline processing at the Chandra X-ray Center (CXC). In the process of converting the level 1 events file to a level 2 events file for each of the observations the following steps were performed: Charge transfer inefficiency (CTI) correction, removal of the pixel randomization, filtering for event grades (retaining the standard ASCA grades 0, 2, 3, 4, and 6), and

\footnotetext{
${ }^{1}$ CIAO is made available by the $\mathrm{CXC}$ and can be downloaded from http://cxc.harvard.edu/ciao/download-ciao-reg.html
}

Table 2. Observing log.

\begin{tabular}{lccrr}
\hline \hline Target & Obs-ID & UT Start [hh:mm:ss] & Expo [ks] \\
\hline G1 569 Bab & 4470 & 2004-06-18 & $05: 59: 44$ & 25.2 \\
HD 130948 BC & 4471 & $2004-11-24$ & $07: 45: 33$ & 42.5 \\
& 6166 & $2004-11-27$ & $09: 22: 07$ & 7.3 \\
HR7329 B & 4472 & 2004-08-06 & $12: 44: 55$ & 47.0 \\
\hline
\end{tabular}

application of the standard good time interval (GTI) file. The events file was also checked for any systematic aspect offsets using CIAO software, but none were present. For HD 130948 the level 2 events files of the two observations were merged for the purpose of source detection. Source detection was carried out with the wavdetect algorithm on an image of $50 \times 50$ pixels length $\left(1\right.$ pixel $\left.=0.492^{\prime \prime}\right)$ centered on the computed position of the primary using wavelet scales between 1 and 8 in steps of $\sqrt{2}$.

As a consequence of the small binary separations of our targets $\left(\sim 2.5-5^{\prime \prime}\right)$, the cross-correlation between X-ray and optical/IR positions requires precise astrometry. We translated the Hipparcos position of the primary star to the time of the Chandra observation using its proper motion (Perryman et al. 1997). The position of the companion relative to the primary was then obtained using the separation and PA given in the literature (see Table 1).

In Fig. 2 we show the corresponding Chandra ACIS images for all three targets. X-ray positions are indicated by the $3 \sigma$ source ellipse of wavdetect, and optical positions are marked by crosses. We label the primary with "A", and the companions with "B". (In two of the targets the companion is itself a binary, composed of two BDs, but with separations below Chandra's resolution limit, cf. Table 1.)

We carefully selected individual photon extraction areas for each component of the three targets. For the BDs circular regions were chosen with radius determined by the separation to the primary and by the X-ray brightness of the primary. Two of the primaries (G1569 A and HD 130948 A) are very strong X-ray emitters, and pile-up is not negligible. For these two sources we use annular photon extraction regions, where the inner radius is chosen such as to minimize the loss of statistics while removing all pile-up (see Sects. 4.2 and Stelzer (2004) for details). The third primary (HR 7329 A) is not detected. The background was selected for each target from carefully defined regions near the X-ray positions. In particular, in the case of HD 130948 BC, which is located in the wings of the PSF of its primary, we extracted the background from an area on the opposite side of and with the same separation from the primary. For undetected objects upper limits to the source counts were computed following the prescription for Poisson distributed data given by Kraft et al. (1991).

For objects that are undetected or too faint for spectral analysis X-ray fluxes are estimated with PIMMS ${ }^{2}$ from the measured count rates assuming an unabsorbed isothermal Raymond-Smith plasma (Raymond \& Smith 1977).

\footnotetext{
2 PIMMS is available at

http://asc.harvard.edu/toolkit/pimms.jsp
} 

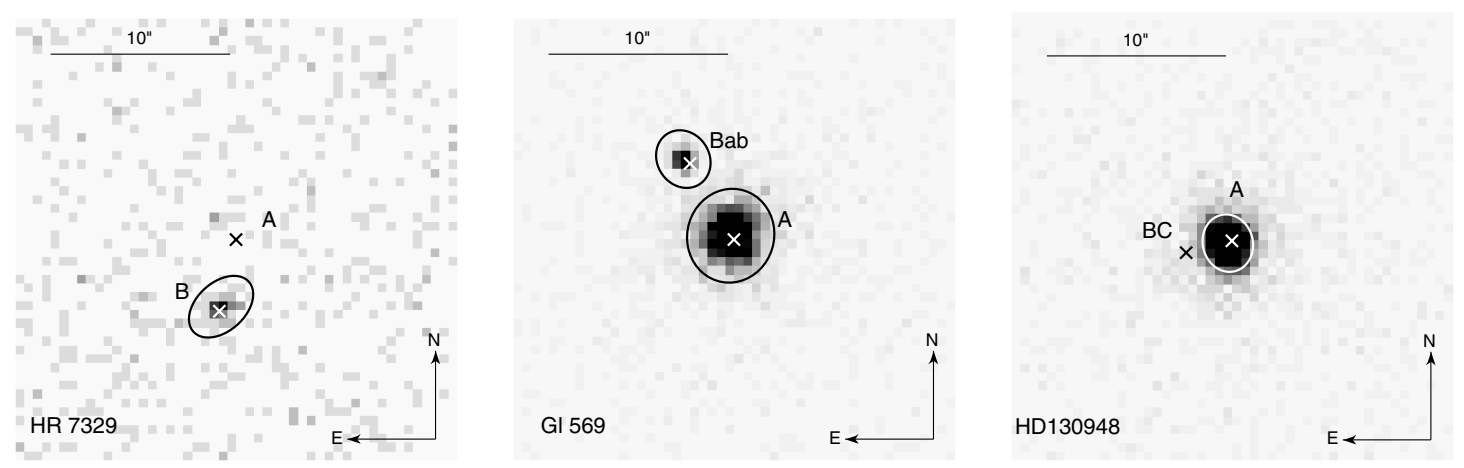

Fig. 2. X-ray images of stellar systems with BD secondary observed with Chandra ACIS-S: left - HR 7329 (12 Myr), middle - Gl 659 (300 Myr), right - HD 130948 (550 Myr). The primaries are labeled " $\mathrm{A}$ ".

Table 3. X-ray parameters of primaries in observed systems.

\begin{tabular}{|c|c|c|c|c|c|}
\hline \multirow[b]{2}{*}{ Name } & \multirow[b]{2}{*}{ SpT } & \multirow[b]{2}{*}{$\log \frac{L_{\mathrm{bol}}}{L_{\odot}}$} & \multicolumn{2}{|c|}{ [0.5-8 keV] } & \multirow[b]{2}{*}{$\left\langle E_{\mathrm{ph}}\right\rangle$} \\
\hline & & & $\begin{array}{c}\log L_{\mathrm{x}} \\
{[\mathrm{erg} / \mathrm{s}]}\end{array}$ & $\log \frac{L_{\mathrm{X}}}{L_{\mathrm{bol}}}$ & \\
\hline HR 7329 A & A0 & +1.37 & $<26.1$ & $<-8.8$ & - \\
\hline Gl 569 A Quies & $\mathrm{dM} 2 \mathrm{e}$ & -1.46 & 28.3 & -3.8 & 0.81 \\
\hline Gl 569 A Flare & $\mathrm{dM} 2 \mathrm{e}$ & -1.46 & 28.7 & -3.4 & 0.90 \\
\hline HD 130948 A & $\mathrm{G} 2$ & +0.13 & 28.8 & -4.9 & 0.81 \\
\hline
\end{tabular}

The counts-to-energy conversion factor is independent of the temperature in the range of $0.3-1 \mathrm{keV}$ which is typical for coronal plasmas of VLM stars and BDs (see also discussion by Audard et al. 2005). Finally, fluxes are converted to luminosities using the Hipparcos distances of the primaries. The X-ray luminosities and X-ray spectral parameters are listed in Table 1 for the BDs and in Table 3 for the primaries. For lack of sufficient statistics we provide the median photon energies for the BDs rather than a coronal temperature derived from spectral modeling. Next we discuss the results on an individual basis.

\section{Results}

\section{1. $H R 7329$}

The Vega-like A0 star HR 7329 was first considered to be a member of the Tucanae association (Zuckerman \& Webb $2000)$, but later shown to belong to the " $\beta$ Pic moving group" (Zuckerman et al. 2001a). This implies that its age has been revised downward (to $12 \mathrm{Myr}$ instead of $\sim 40 \mathrm{Myr}$ ).

HR 7329B, at a separation of $4.2^{\prime \prime}$ from the A-type star, was discovered by Lowrance et al. (2000) and a spectral type M7.5 V was assigned, corresponding to substellar mass under the assumption that it forms a bound pair with HR 7329 A. It was indeed established by Guenther et al. (2001) that both objects share common proper motion.

In the Chandra image there is only one X-ray source at the position of the binary, coinciding with the BD. The total number of source photons collected from HR 7329 B is 66.5 counts, after subtracting the background, resulting in a time-averaged luminosity of $\log L_{\mathrm{x}}=27.2 \mathrm{erg} / \mathrm{s}$. The $\log \left(L_{\mathrm{x}} / L_{\mathrm{bol}}\right)$ ratio is -3.8 , within the typical range of late-type stars. The spectral
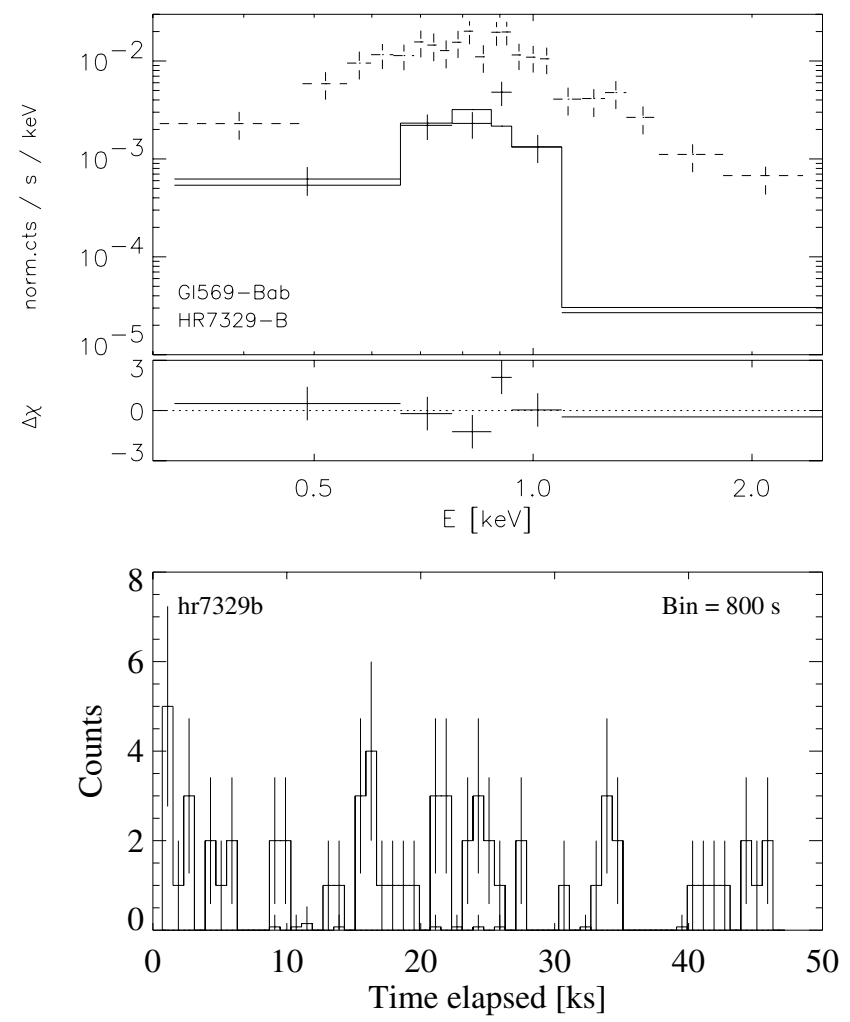

Fig. 3. Top: Chandra spectrum of HR 7329 B, best fit 1-T APEC model, and residuals. The dashed lines represent the spectrum of Gl 569 Bab (see Stelzer 2004), shown for comparison. Bottom: Chandra lightcurve of HR 7329 B. The background, also shown and scaled to the source extraction area, is negligible.

shape is not well constrained due to the poor statistics. In the top panel of Fig. 3 we show the X-ray spectrum of HR 7329 B overlaid by the best fit of a one-temperature (1-T) APEC 3 model with subsolar abundances $\left(Z=0.3 Z_{\odot}\right)$. No photoabsorption term is required to fit the spectrum. The temperature of the best fit model is $0.49 \mathrm{keV}(=5.7 \mathrm{MK})$.

\footnotetext{
${ }^{3}$ For spectral fitting we use the Astrophysical Plasma Emission Code within the XSPEC environment version 11.3.0; see Smith et al. (2001) for a description of APEC.
} 
The lightcurve of HR 7329 B (bottom panel of Fig. 3) is suggestive of variability. A KS-test applied to the photon arrival times yields a probability of $97 \%$ for variability. Furthermore, we used the MLB algorithm, a method to determine periods of constant signal from a list of photon arrival times, based on a maximum likelihood algorithm under the assumption of Poisson statistics; see Wolk et al. (2005) for a description of this technique. However, no change point is found at significance $\geq 95 \%$, i.e. the whole lightcurve can be described by constant count rate.

The A0 star HR 7329 A is X-ray dark down to the detection limit, with an upper limit of $\log L_{\mathrm{x}}<26.1 \mathrm{erg} / \mathrm{s}$, corresponding to $\log \left(L_{\mathrm{X}} / L_{\mathrm{bol}}\right)<-8.8$. This is (one of the) most sensitive upper limits for the X-ray emission from an A-star. It is by three (!) orders of magnitude more sensitive than the upper limit derived from the ROSAT All-Sky Survey for the combined system HR 7329 AB (Stelzer \& Neuhäuser 2000).

\section{2. $H D 130948$}

HD $130948 \mathrm{~A}$ is a young solar analog (spectral type G2 V). A comparatively young age $(0.3-0.8 \mathrm{Gyr})$ is indicated by high lithium abundance, chromospheric and coronal activity, and fast rotation (Gaidos 1998).

Two cool companions were discovered by Potter et al. (2002). The two objects, HD 130948 B and C most likely form a gravitationally bound binary, and they both have spectral type L4 (Potter et al. 2002; Goto et al. 2002). HD 130948 B and $\mathrm{C}$ are unresolved with Chandra (separation $0.13^{\prime \prime}$ ), and separated from the primary by only $2.6^{\prime \prime}$. Figure 2 shows that HD 130948 BC is situated in the outer wing of the PSF of the primary. There is no obvious enhancement of the count rate at the position of HD $130948 \mathrm{BC}$ with respect to other locations with the same distance to HD 130948 A. To examine the area in more detail we have deconvolved the ACIS image with the PSF using the IDL task MAX_LIKELIHOOD ${ }^{4}$. No additional source is seen on the resulting reconstructed image at the position of HD $130948 \mathrm{BC}$, and we conclude that the BD pair is not an X-ray emitter down to the sensitivity limit of this Chandra observation. We derive an upper limit of $\log L_{\mathrm{X}}<25.6 \mathrm{erg} / \mathrm{s}$. In terms of $\log \left(L_{\mathrm{x}} / L_{\mathrm{bol}}\right)$ the upper limit is $-4.1 /-4.2$, depending on whether it is attributed entirely to HD $130948 \mathrm{~B}$ or C.

The primary HD $130948 \mathrm{~A}$ is a bright X-ray source with roughly $0.2 \mathrm{cts} / \mathrm{s}$ in the $0.5-8 \mathrm{keV}$ band, corresponding to a pile-up fraction of $\sim 20 \%$ according to Fig. 6.18 of the Chandra Proposers' Observatory Guide ${ }^{5}$. The negative effects of pile-up (distortion of the shape of the lightcurve and spectrum and underestimated flux) can be avoided by using an annular photon extraction region avoiding the core of the PSF, obviously at the expense of statistics.

For the spectral analysis of HD 130948 A we consider only the longer observation, Obs-ID 4471. To determine the smallest inner radius $r_{\text {in }}$ for an annular source area that reduces pile-up

\footnotetext{
${ }^{4}$ MAX_LIKELIHOOD.PRO is a tool provided by the IDL Astronomy User's Library, see

http://idlastro.gsfc.nasa.gov/homepage.html

${ }^{5}$ http://cxc.harvard.edu/proposer/POG/index.html
}
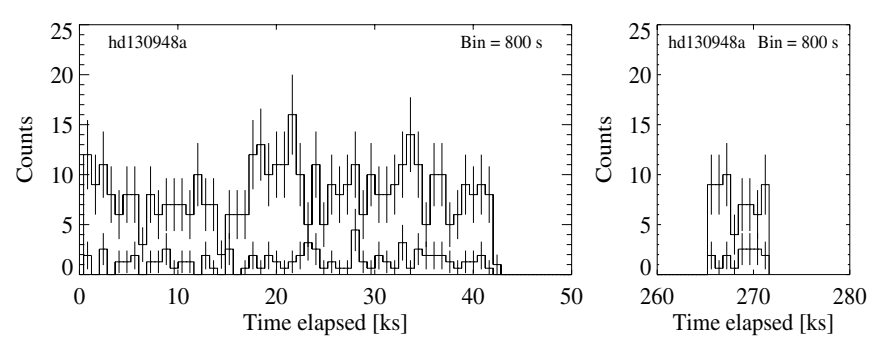

Fig. 4. X-ray lightcurve of HD 130948 A and background; source photons were extracted from an annulus with inner radius of $1.5^{\prime \prime}$, thus avoiding the central portion of the PSF which is subject to pile-up.

without losing too much statistics we extracted events from a series of annuli with $r_{\text {in }}=0.5^{\prime \prime} \ldots 3.75^{\prime \prime}$ and outer radius fixed at $8^{\prime \prime}$. As discussed above no significant contribution is present from the BD pair, and there are also no other X-ray sources in the extraction region of HD 130948 BC. With help of the $\mathrm{ChaRT}^{6}$ and $\mathrm{MARX}^{7}$ simulators an individual arf was constructed for each of the annuli to correct the effective area for the missing part of the $\mathrm{PSF}^{8}$. The X-ray luminosity computed from the individual spectra increases as a function of $r_{\text {in }}$ until it reaches a plateau when all of the piled-up portion of the PSF is excluded. The kink in the distribution $L_{\mathrm{x}}-r_{\text {in }}$ defines the optimized photon extraction radius. From this plateau we determine a luminosity of $\log L_{\mathrm{x}}=28.79 \pm 0.05 \mathrm{erg} / \mathrm{s}$. This is in reasonable agreement with the measurement by Hünsch et al. (1999) of $\log L_{\mathrm{x}}=29.0 \mathrm{erg} / \mathrm{s}$ based on the ROSAT All-Sky Survey, considering the known variability of $L_{\mathrm{x}}$ in solar-like stars and the different characteristics of the satellites. The X-ray luminosity of HD $130948 \mathrm{~A}$ is also consistent with expectations for main-sequence stars of similar rotation periods $\left(P_{\text {rot }}=\right.$ 7.85 d; Gaidos 2000) and age; see e.g. Fig. 3 in Pizzolato et al. (2003). The spectrum extracted from the optimized region can be described with an iso-thermal APEC model of $k T \sim 0.5 \mathrm{keV}$ $(\log T[\mathrm{~K}]=6.76=5.8 \mathrm{MK})$ and subsolar global abundances of $Z=0.5 Z_{\odot}$. The Chandra lightcurve of HD 130948 A shows variability on timescales of hours, but no strong flaring (Fig. 4).

\subsection{GI569}

This is the only of the three targets where both the primary and the BD companion(s) are detected (see Fig. 2). The data analysis and the results for G1 $569 \mathrm{Bab}$ have been described in detail by Stelzer (2004). We summarize the results for the BD pair in Table 1.

G1 569 A suffers from pile-up. We analysed its spectrum in an analogous way to that of HD 130948 A. The large flare that occurred $\sim 5 \mathrm{ks}$ after the start of the observation (see Fig. 2 in Stelzer 2004) is eliminated from the quiescent spectrum and its spectrum is analysed separately. The mean X-ray luminosity

\footnotetext{
6 Information on the usage of the Chandra Ray Tracer (ChaRT) can be found at http://cxc. harvard. edu/chart/

7 The Model of AXAF Response to X-rays (MARX) is provided by the MIT/CXC (see http://space.mit.edu/CXC/MARX/)

8 See http://www.astro.psu.edu/users/tsujimot/ arfcorr.html and Getman et al. (2005) for more information on the procedure.
} 
(averaged over the full observation) is in accordance with the ROSAT result (Hünsch et al. 1999). During the flare the temperature increased slightly from $k T_{\mathrm{Q}}=0.81 \mathrm{keV}(\lg T=6.97$; $9.4 \mathrm{MK})$ to $k T_{\mathrm{F}}=0.90 \mathrm{keV}(\lg T=7.02,10.4 \mathrm{MK})$, as expected if additional heating is taking place during the outburst.

\section{Discussion}

\subsection{X-ray properties of brown dwarfs}

For the two detected BDs from our sample, HR 7329 B and G1 $569 \mathrm{Bab}$, the X-ray spectra are rather similar. This is remarkable because HR 7329 B is a relatively young BD with lowamplitude variability, while Gl 569 Bab is relatively old and has shown a strong flare during the Chandra observation.

Detailed characterizations of the X-ray spectra of BDs have remained out of reach to date due to poor statistics: all observations undertaken so far have yielded a few dozen counts per source at most. Therefore, when comparing our sample to previous observations of BDs, we consider the median photon energy $\langle E\rangle$ as representative for the temperature of the emitting plasma. Comparing the time-averaged $\langle E\rangle$ we notice again the similarity of HR $7329 \mathrm{~B}$ and Gl $569 \mathrm{Bab}$. When computing $\langle E\rangle$ separately for the flare and quiescent times of Gl $569 \mathrm{Bab}$ it is seen that (i) during quiescence Gl $569 \mathrm{Bab}$ is softer than HR 7329 B, and (ii) the time-average of G1 $569 \mathrm{Bab}$ is dominated by the flare. The values of $\langle E\rangle$ for our BD targets are somewhat lower than for the younger BDs in IC 348 (see Fig. 5 of Preibisch \& Zinnecker 2002), suggesting a softer X-ray spectrum. However, one must keep in mind that the X-ray spectrum of young BDs suffers from extinction. The X-ray detected BDs in IC 348 have a range of $A_{\mathrm{J}}=$ $0.2 \ldots 1.8 \mathrm{mag}$, with the majority of them at $A_{\mathrm{J}} \sim 0.4 \mathrm{mag}$, corresponding to $A_{\mathrm{V}} \sim 1.4 \mathrm{mag}$ and $N_{\mathrm{H}} \sim 0.3 \times 10^{22} \mathrm{~cm}^{-2}$. Similarly, Preibisch et al. (2005a) found $\langle E\rangle \sim 1 . . .2 \mathrm{keV}$ for BDs in the ONC. These BDs are absorbed by $A_{\mathrm{V}}=1.4 \ldots .6 .8 \mathrm{mag}$.

We have simulated spectra with XSPEC on a grid of $k T$ and $N_{\mathrm{H}}$ values, and computed $\langle E\rangle$ for each of the theoretical spectra. In Fig. 5 we show the resulting relation between the median photon energy and the temperature of the 1-T model for different values of $N_{\mathrm{H}}$. If for a given BD the absorption is known the X-ray temperature can be estimated from $\langle E\rangle$. We use the only two BDs for which both $\langle E\rangle$ and $k T$ have been measured, G1 $569 \mathrm{Bab}$ and HR $7329 \mathrm{~B}$, as a cross-check of this procedure, and, indeed, we obtain $k T$ compatible with the results from spectral fitting of the data; compare Fig. 5 to text in Sect. 4. We estimate in the same way $k T$ for the BDs in the ONC using extinction and median photon energies given by Preibisch et al. (2005a). Figure 5 shows that for these objects the rather small range of measured $\langle E\rangle$ corresponds to a large spread in $k T$, because of their wide range of absorption. Some of the very young BDs in the ONC apparently have soft spectra like the more evolved BDs we discuss in this paper. This is unlike in stars, where X-ray temperature decreases with increasing age (Favata \& Micela 2003). However, some other ONC BDs are much harder. Unfortunately, the ONC BD sample is to our knowledge the only one for which the parameter $\langle E\rangle$ was presented, and therefore at this point we can not

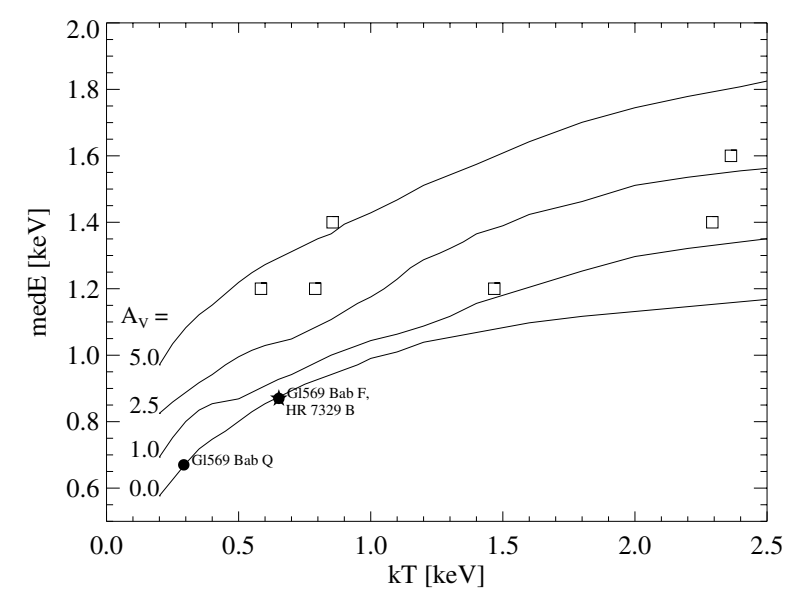

Fig. 5. Median photon energy vs. X-ray temperature obtained from simulations of a 1-T APEC spectrum with photo-absorption. The individual curves correspond to spectra with different column density $N_{\mathrm{H}}$. We assumed a standard extinction law of $N_{\mathrm{H}}\left[\mathrm{cm}^{-2}\right]=2.0 \times$ $10^{21} A_{V}$ [mag] (Ryter 1996). Squares denote BDs in the ONC from Preibisch et al. (2005a).

draw firm conclusions on the evolution of coronal temperature with age in BDs.

Our third target, HD 130948 BC, is undetected with an upper limit to $L_{\mathrm{x}}$ three orders of magnitude lower than the luminosity determined for its primary HD $130948 \mathrm{~A}$. We mention in passing that Tsuboi et al. (2003) have studied a similar case: TWA-5 B, which was detected and resolved with Chandra from its primary. The two components in that system have an even smaller separation of 2 ". But TWA-5 A is "only" two orders of magnitude brighter in X-rays than the $\mathrm{BD}$, and TWA-5 B has an at least $\sim 10$ times larger flux than HD 130948 B.

\subsection{BD X-ray emission and stellar parameters}

The new Chandra observations put us for the first time in the position to study the X-ray properties of BDs in restricted ranges of mass and temperature. The parameter space that can be studied on basis of the existing data is high-lighted by grey shades in Fig. 1: the mass range $0.05-0.07 M_{\odot}$ and the $T_{\text {eff }}$ range $2400-2800 \mathrm{~K}$.

When comparing X-ray luminosities for BDs obtained from different observations, we must take into account that different authors have used different energy bands for the evalution of $L_{\mathrm{x}}$. We have used PIMMS to convert all $L_{\mathrm{x}}$ values from the literature to the $0.5-8 \mathrm{keV}$ band. The assumed spectral form is a Raymond-Smith model with individual temperature based on the results presented in the literature with the exception of DENIS J1556. Bouy (2004) has assumed an X-ray temperature of $2 \mathrm{keV}$ for this BD in Upper Sco, but we suspect that the actual temperature might be substantially lower, such as observed on TWA-5 B and on HR 7329 B which are only slightly older than DENIS J1556. If the temperature of DENIS J1556 is $0.4 \mathrm{keV}$ the $\mathrm{X}$-ray luminosity decreases by a factor of 0.7 with respect to the value for $2 \mathrm{keV}$. In this study, instead of the published value, we use $\log L_{\mathrm{x}}=28.1 \mathrm{erg} / \mathrm{s}$ for DENIS J1556, derived from a recent (re-)analysis of the same XMM-Newton 

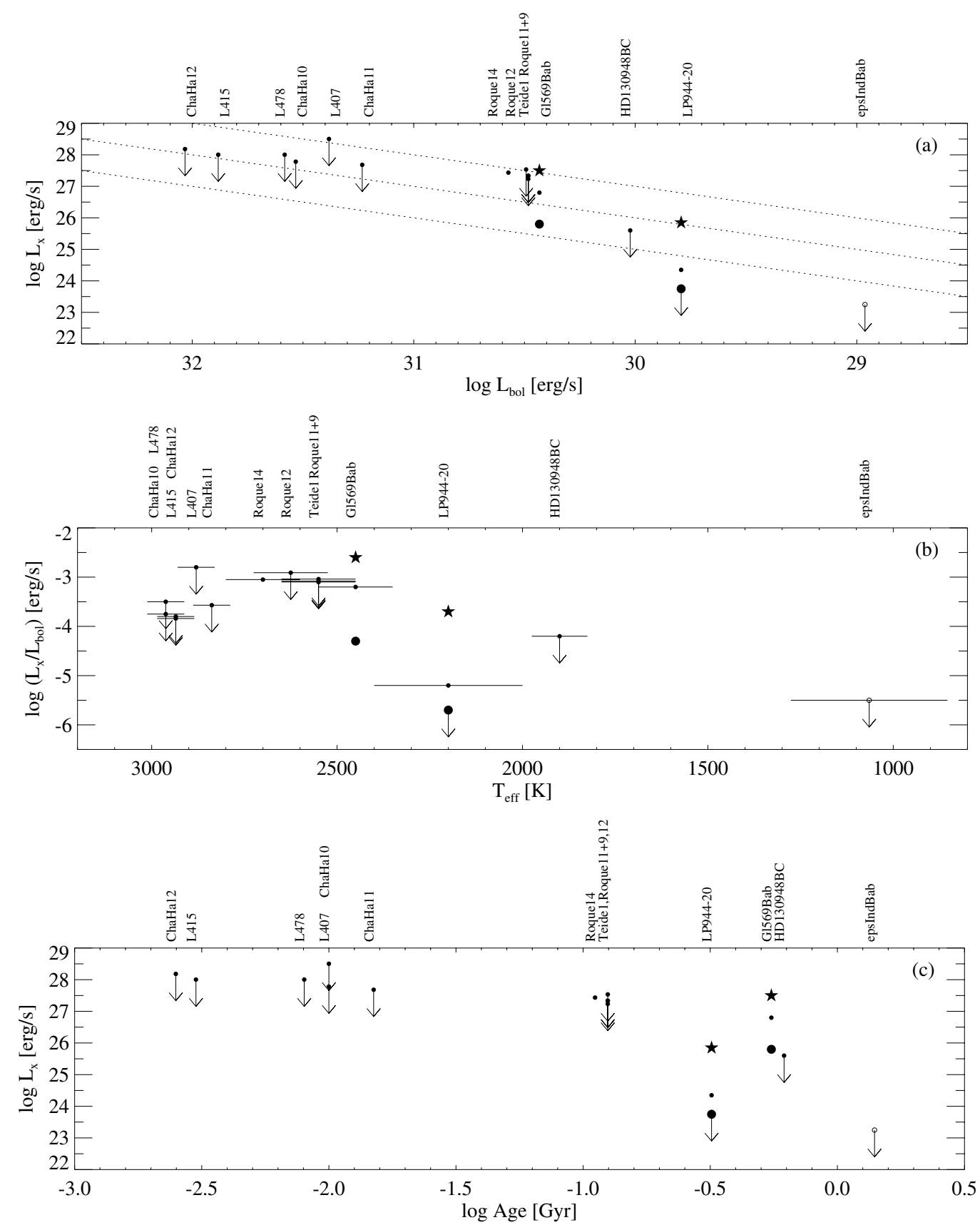

Fig. 6. X-ray activity of BDs with $M \sim 0.05-0.07 M_{\odot}$ versus $L_{\mathrm{bol}}$ a), versus $T_{\mathrm{eff}}$ b) and versus age c). Plotting symbols: small circles - Timeaveraged $L_{\mathrm{x}}$ from Chandra and XMM-Newton observations; star symbols $-L_{\mathrm{x}}$ in the flare peak; large circles - Quiescent $L_{\mathrm{x}}$. Dotted lines in the uppermost panel indicate $L_{\mathrm{x}} / L_{\mathrm{bol}}$ levels of $10^{-3}, 10^{-4}$, and $10^{-5}$.

data (Argiroffi et al., in prep.). This is smaller by a factor 4 than the estimate by Bouy (2004), and yields a $\log \left(L_{\mathrm{x}} / L_{\mathrm{bol}}\right)$ ratio of -3.3 , more typical for coronal emission than the published value of -2.69 .

\subsubsection{High-mass brown dwarfs $\left(0.05-0.07 M_{\odot}\right)$}

First we examine how the X-ray emission of BDs with the same mass $\left(0.05-0.07 M_{\odot}\right.$; shaded dark grey in Fig. 1) is related to bolometric luminosity and to effective temperature. We have included $\epsilon$ Ind Bab in this "high-mass" BD sample because it is the only object with sensitive X-ray data that allows us to extend the temperature range significantly below $2000 \mathrm{~K}$. But let's keep in mind that the masses of both components in this BD binary are slightly lower than the lower threshold of the studied mass bin $\left(M_{\epsilon \mathrm{IndBa}} \sim 0.045 M_{\odot} ; M_{\epsilon \mathrm{IndBb}} \sim 0.025 M_{\odot}\right.$; McCaughrean et al. 2004). Therefore, $\epsilon$ Ind Bab is shown with an open plotting symbol in Fig. 6.

Figure 6 a shows the relation between $L_{\mathrm{x}}$ and $L_{\mathrm{bol}}$ for the sample of $0.05-0.07 M_{\odot}$ objects. For unresolved binaries we have summed up the bolometric luminosities of both components. Most data points in Fig. 6a fall into the $10^{-3 \ldots-5}$ range typical for late-type active stars. But the slope seems to steepen for the least luminous objects which correspond to older ages. 

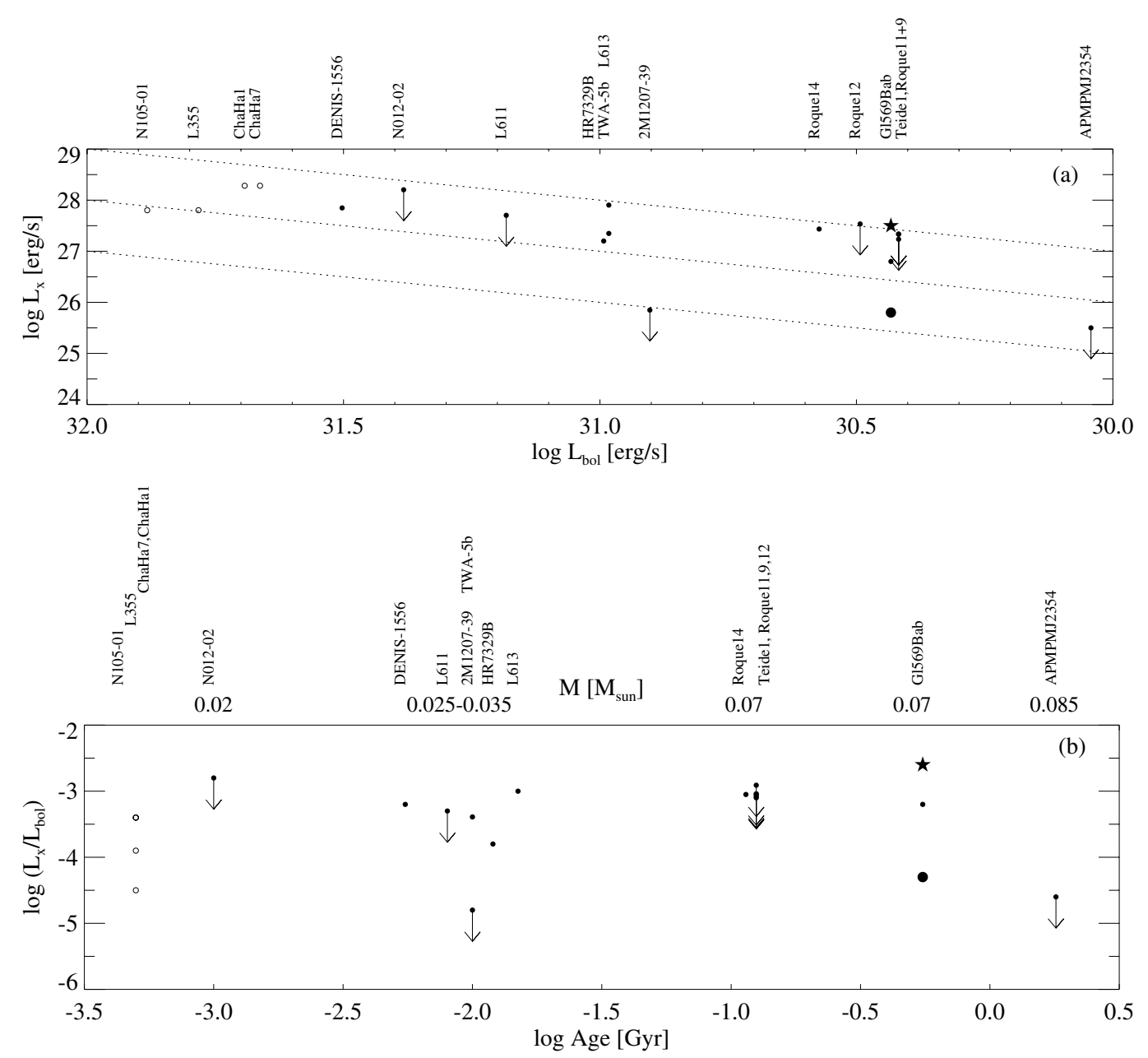

Fig. 7. X-ray activity for BDs with $T_{\mathrm{eff}}=2400 \ldots 2800 \mathrm{~K}$ : top $-L_{\mathrm{x}}$ versus $L_{\mathrm{bol}}$, bottom $-L_{\mathrm{x}} / L_{\mathrm{bol}}$ ratio versus age. BDs above the Baraffe et al. (1998) and Chabrier et al. (2000) models in the HR diagram are arbitrarily placed at an age of $0.5 \mathrm{Myr}$, and given open plotting symbols.

In other words, there is a decline of the $L_{\mathrm{x}} / L_{\mathrm{bol}}$ ratio as the BDs evolve; see Fig. 6c discussed in more detail below.

Figure $6 \mathrm{~b}$ shows the $L_{\mathrm{x}} / L_{\mathrm{bol}}$ ratio as a function of $T_{\text {eff }}$ for the same sample. Despite the large number of upper limits, there is evidence for a decline of the fractional X-ray luminosity $\left(L_{\mathrm{x}} / L_{\mathrm{bol}}\right)$ with $T_{\text {eff }}$. This underlines that the atmospheric temperature plays a crucial role in determining the level of X-ray activity of evolved BDs. Currently, there are only two data points below $T_{\text {eff }} \sim 2000 \mathrm{~K}$, i.e. in the regime of the $\mathrm{L}$ and $\mathrm{T}$ dwarfs, and both are non-detections. Given that the data set is dominated by upper limits, we have refrained from a more detailed analysis involving statistical tests.

From Figs. $6 \mathrm{a}$ and $6 \mathrm{~b}$ it strikes that none of the IC 348 and Cha I BDs in the $0.05-0.07 M_{\odot}$ mass bin was detected in $\mathrm{X}$-rays. As can be seen from Fig. 1 all but one of the X-ray detected objects from the IC 348 and Cha I sample have either masses above the substellar limit or they are extremely young (above the model grids), when compared to Baraffe et al. (1998) and Chabrier et al. (2000) models. On the other hand, four of the six IC 348 and Cha I members in our 0.05-0.07 $M_{\odot}$ bin have ages of $\sim 10 \mathrm{Myr}$. This can be seen from Fig. $6 \mathrm{c}$, which shows the evolution of X-ray luminosity with age.
The observed $L_{\mathrm{x}} / L_{\mathrm{bol}}$ values of detected young BDs at similar $T_{\text {eff }}$ but lower or higher mass than the range examined in Fig. 6 are comparable to the observed upper limits, such that the emission levels of the undetected young BDs in the 0.05-0.07 $M_{\odot}$ bin are expected to be only little below their upper limits. Therefore, even a wrong assignment of masses for the young BDs by the evolutionary models - e.g. the D'Antona \& Mazzitelli (1997) models assign systematically lower masses for BDs of given $T_{\text {eff }}$ than the Baraffe et al. (1998) and Chabrier et al. (2000) models - does not change our consideration.

\subsubsection{Hot Brown dwarfs ( $T_{\text {eff }}=2400-2800 \mathrm{~K}$ )}

The dependence of X-ray luminosity on $L_{\mathrm{bol}}$ for BDs in a given range of $T_{\text {eff }}(2400-2800 \mathrm{~K}$; see light grey shade in Fig. 1) is displayed in Fig. 7a. Most BDs fall in a narrow band defined by $L_{\mathrm{x}} / L_{\mathrm{bol}}=10^{-3 \ldots-4}$, indicating that the X-ray activity levels for "hot" BDs are (A) similar irrespective of age and mass, and (B) near the upper end of the canonical values for late-type stars.

The dependence of X-ray activity on age for the same $T_{\text {eff }}$ bin is examined in Fig. 7b. Here, the most notable feature is the low upper limit of 2M 1207-39, which deviates by nearly 


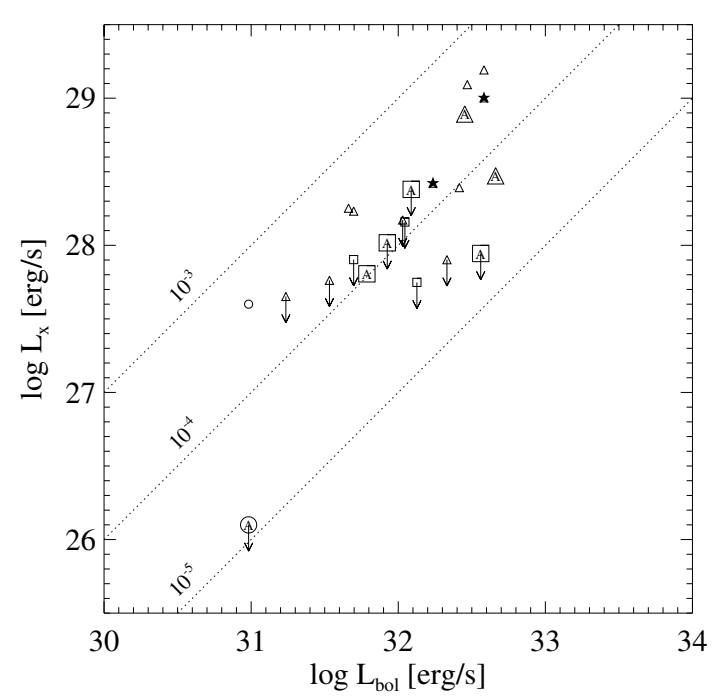

Fig. 8. Ratio of $\log L_{\mathrm{x}}$ versus $\log L_{\text {bol }}$ for young BDs and BD candidates (spectral type M 5 and later): triangles, squares, open circles BDs in Cha I, IC 348, TW Hya. The plot includes objects with spectral type M 5 and later with published measurement of $\mathrm{H} \alpha_{10}$. Accretors are marked with "A" and a larger plotting symbol. Two objects in Cha I that have flared during the X-ray observation are marked with an asterisk.

two orders of magnitude from the other BDs at the same age of $\sim 10 \mathrm{Myr}$. Note, that all objects in this age range have similar mass of $\sim 0.025-0.035 M_{\odot}$. Accretion may be an additional parameter that influences the observed X-ray emission in young BDs. Only two BDs among the objects shown in Fig. 7 display signatures for accretion, 2M 1207-39 and L355; see discussion in Sect. 5.3.

\subsection{BD X-ray emission and accretion}

In Fig. 8 we show only the young BDs (IC 348, Cha I, TW Hya) in the $L_{\mathrm{x}}-L_{\mathrm{bol}}$ diagram with the purpose of examining the influence of accretion on X-ray emission from BDs. We distinguish accreting from non-accreting objects by a full width at $10 \%$ of the peak flux of $\mathrm{H} \alpha\left(\mathrm{H} \alpha_{10}\right)$ larger than $200 \AA$; following Jayawardhana et al. (2003). This requires that the sample is restricted to BDs with measurements of $\mathrm{H} \alpha_{10}$. The $\mathrm{H} \alpha$ data is taken from the literature (Jayawardhana et al. 2003, Natta et al. 2004, Muzerolle et al. 2005 and Mohanty et al. 2005 for the star forming regions, and Mohanty et al. 2003 for TWA). It turns out that $\mathrm{H} \alpha_{10}$ is available for only 7 out of 19 young bona-fide BDs, and only two of them (L 355 in IC 348 and 2M 1207-39 in TWA) are identified as accreting.

A broader statistical basis is obtained when including BD candidates, i.e. objects with spectral type M 5 to M7. In this sample 22 objects have both sensitive X-ray measurements and $\mathrm{H} \alpha_{10}$ data, and 7 are above the $200 \AA$ threshold for accretion. The accretors are identified by an "A" surrounded by a larger symbol in Fig. 8. There may be some trend for the accretors to show lower $L_{\mathrm{x}} / L_{\mathrm{bol}}$ ratio than the non-accreting young BDs, but admittedly this assertion needs to be corroborated by a much larger sample. We deem worth noting, however, that the two BDs in TW Hya behave very differently both in X-rays and

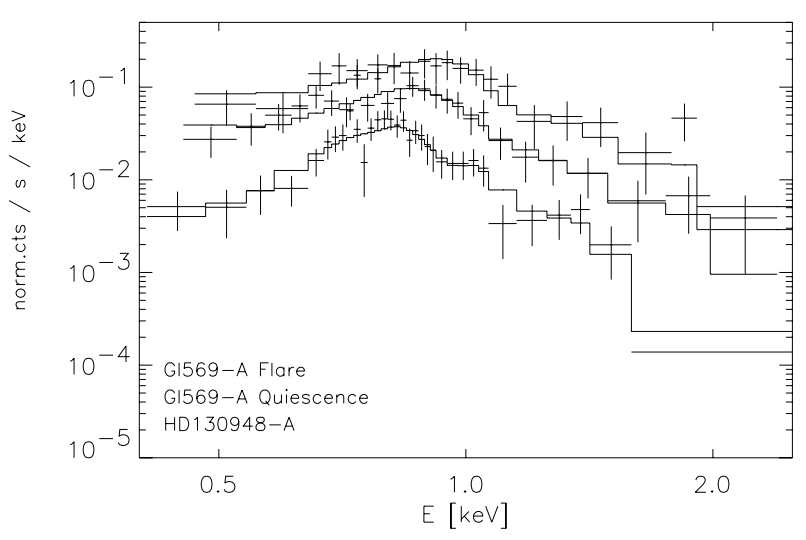

Fig. 9. Spectra of the primaries, from top to bottom Gl 569 A during flare phase, Gl 569 A during quiescence, and average of HD 130948 A. The spectra of Gl 569 A are offset in the vertical direction for clarity. The enhanced bin at $\sim 1.85 \mathrm{keV}$ in the flare spectrum of Gl 569 A may point at strong Si XIII emission.

in $\mathrm{H} \alpha$ : TWA-5 B is a rather bright $\mathrm{X}$-ray source with moderate $\mathrm{H} \alpha$ emission $\left(W_{\mathrm{H} \alpha}=5 \AA, \mathrm{H} \alpha_{10}=162 \AA\right.$; Mohanty et al. 2003), while $2 \mathrm{M} 1207-39$ is undetected in X-rays down to a rather sensitive upper limit (Gizis \& Bharat 2004), but is known to exhibit broad and strong $\mathrm{H} \alpha$ emission $\left(W_{\mathrm{H} \alpha}=28 \AA, \mathrm{H} \alpha_{10}=204 \AA\right.$; Mohanty et al. 2003).

The $\mathrm{H} \alpha$ profile of $2 \mathrm{M} 1207-39$ was recently shown to be strongly variable with $10 \%$ width ranging from $200 \ldots 300 \AA$ (Scholz et al. 2005). This leads us to caution that the number of accretors in our sample might actually be a lower limit, and one or more of the BDs below the $200 \AA$ threshold may have been caught in a temporary low-accretion phase during the $\mathrm{H} \alpha$ observations. Furthermore, for an ideal comparison X-ray and optical data should be obtained simultaneously. However, the X-ray emission of young BDs (and also that of higher-mass $\mathrm{T}$ Tauri stars), undergoes only modest changes on a long time baseline (e.g. Stelzer et al. 2004). Therefore, we consider the present data set as representative for young BDs and speculate that accretion may suppress X-ray emission in BDs, similar to what is observed in higher-mass T Tauri stars (see e.g. Stelzer \& Neuhäuser 2001; Flaccomio et al. 2003). Several mechanisms by which accretion may cause a decrease of X-ray emission in T Tauri stars have been suggested, including distortion of the magnetosphere, increased coronal density, and changes in the interior structure that influence the dynamo process (see Preibisch et al. 2005b).

\subsection{X-ray properties of the primaries}

The nature of the primary was not a selection criterion for the targets of this study, except for their age. Therefore it is not a surprise that they are very different from each other. It is nevertheless interesting to examine and compare their X-ray properties. The ACIS spectra of all detected primaries of our sample are shown in Fig. 9, and the derived X-ray parameters are summarized in Table 3. As a consequence of the special photon extraction procedure required to avoid pile-up, each of the spectra of the detected primaries has only $\sim 500$ counts, such 
that a detailed analysis of their temperature and emission measure distribution is not feasible.

Nevertheless, the temperatures from the iso-thermal fits can be considered as an average coronal temperature, and be compared to the recent work by Telleschi et al. (2005). Their study of solar-analogs is based on high-resolution X-ray spectra, and they defined a mean coronal temperature as the average of the temperatures from the emission measure distribution weighted by the emission measure. In order to compare these different data, we must take into account that different energy bands were used to measure the X-ray luminosities: $0.5-8 \mathrm{keV}$ in our case versus $0.1-10 \mathrm{keV}$ by Telleschi et al. (2005). We verified with PIMMS that this amounts to a flux change by a factor of two for a $1 \mathrm{keV}$ source. After adapting the relation between $T_{\mathrm{x}}$ and $L_{\mathrm{x}}$ observed by Telleschi et al. (2005) for a sample of 6 solar-analogs to the $0.5-8.0 \mathrm{keV}$ band, the predicted X-ray luminosity for HD $130948 \mathrm{~A}$ is $10^{29.0} \mathrm{erg} / \mathrm{s}$, versus the observed value of $10^{28.8} \mathrm{erg} / \mathrm{s}$. Therefore, the G2 star HD $130948 \mathrm{~A}$ behaves like a typical solar-analog, providing further support for the tight relation between $L_{\mathrm{x}}$ and $T_{\mathrm{x}}$ in similar stars. G1569 A, on the other hand, is a much cooler, lower-mass flare star (spectral type dM2e), and, as expected, clearly does not conform to the correlation observed for the $\mathrm{G}$ stars.

The non-detection of HR 7329 A is in agreement with the common understanding that intermediate-mass stars can sustain neither coronal nor wind-driven X-ray emission. Contrary to this expectation, many A-type stars have been detected in $\mathrm{X}$-ray observations with various satellites. However, most of these observations were carried out with instruments with low spatial resolution and cannot exclude the likelihood that the observed emission is to be attributed to nearby unresolved companions (as proven here in the case of HR 7329), or unrelated nearby objects. The very low upper limit we establish for HR $7329 \mathrm{~A}$ is a strong constraint for any activity resulting from eventual core dynamo action (MacGregor \& Cassinelli 2003).

\section{Conclusions}

Our Chandra targets, combined with data from the literature, represent a so far unique sample that has allowed us to make a first quantitative study of the dependence of sub-stellar X-ray emission on critical parameters.

From Fig. 7 it appears to emerge that BDs in young associations ( $\beta$ Pic; TWA), open clusters (Pleiades) and the solar neighborhood (Gl $569 \mathrm{Bab}$ ) that share the same temperature (2400-2800 K) have very similar fractional X-ray luminosities $\left(\log \left(L_{\mathrm{X}} / L_{\mathrm{bol}}\right) \sim 10^{-3 \ldots-4}\right)$, i.e. the X-ray luminosity scales with the bolometric luminosity for "hot" BDs.

Note, that for the older objects this activity level corresponds to higher mass. This phenomenon can probably be extended to the coolest stars above the substellar boundary: MS stars with $T_{\text {eff }}$ in the range examined in Fig. 7, i.e. stars with spectral types later than $\sim \mathrm{M} 7$, show $\log \left(L_{\mathrm{x}} / L_{\text {bol }}\right)$ levels similar to our "hot" BD sample (see e.g. Fig. 4 of Stelzer 2004), and - in general - such stars are thought to have mature ages of a few Gyr. But, strictly speaking, they can not be included in our study because actual age determinations are not available.
However, in the sample restricted to high-mass BDs $\left(0.05-0.07 M_{\odot}\right.$; the only substellar mass range covered so far with sensitive X-ray observations) $L_{\mathrm{x}}$ seems to decrease with $L_{\mathrm{bol}}$ steeper than expected for the canonical $\log \left(L_{\mathrm{x}} / L_{\text {bol }}\right)$ ratio of late-type stars $\left(\sim 10^{-3 \ldots-5}\right)$. This sample includes BDs with low atmospheric temperature. And, indeed, an interesting fall of the $L_{\mathrm{x}} / L_{\mathrm{bol}}$ ratio with $T_{\text {eff }}$ is observed (Fig. 6b).

With the present data it is not possible to unambiguously distinguish the influence of $L_{\mathrm{bol}}$ and $T_{\text {eff }}$ on the X-ray activity level. But we have presented first evidence for a direct correlation between $L_{\mathrm{x}} / L_{\mathrm{bol}}$ and $T_{\mathrm{eff}}$. Such a correlation supports conclusions based on calculations of magnetic diffusivity. Mohanty et al. (2002) found that the high electrical resistivities in the predominantly neutral atmospheres of ultracool dwarfs prevent significant dynamo action by (a) reducing the amount of magnetic stress created by footpoint motions and (b) impeding their efficient transport through the atmosphere. In this vein, improved statistics are required for the critical temperature range, i.e. the regime of late-M and $\mathrm{L}$ dwarfs.

HD $130948 \mathrm{BC}$ is the only X-ray observed L dwarf with known age, and probably the youngest one. Unfortunately, its upper limit is rather high due to contamination with photons of the nearby X-ray bright primary. Together with all other L dwarfs studied with reasonable sensitivity (Stelzer \& Neuhäuser 2002; Berger et al. 2005) it is undetected, such that no $\mathrm{L}$ dwarf has been detected in X-rays so far. The situation is thus qualitatively similar to what is found for chromospheric $\mathrm{H} \alpha$ activity: $\mathrm{H} \alpha$ emission shows a sharp drop at the latest $M$ types, albeit still a significant fraction of early $L$ dwarfs has $\mathrm{H} \alpha$ in emission (Gizis et al. 2000; Mohanty \& Basri 2003). But this apparent discrepancy to the X-ray observations might be due to the much larger data base for chromospheric emission in ultracool dwarfs as opposed to coronal emission.

We examined the incidence of accretors in the X-ray sample of young BDs, and find marginal evidence that accreting BDs have lower X-ray luminosity than non-accreting ones. If confirmed by future studies on a larger sample this would show that activity on BDs is subject to the same mechanisms that suppress X-ray emission in pre-MS stars during their T Tauri phase.

With the presentation of two X-ray spectra for a sample of three evolved BDs observed with Chandra we have demonstrated the high potential of this X-ray satellite for the study of substellar coronae. Similar values for X-ray temperature are measured for the 12 Myr old HR 7329 B during a relatively quiescent state and for the $200-400$ Myr old Gl 569 Bab during a large flare. A systematic study of temperatures of the X-ray emitting plasma in BDs is impeded by the absence of similarly high-quality data in the previous X-ray literature. But we have shown that the median photon energy can be used to infer the $\mathrm{X}$-ray temperature of the corresponding spectrum, if the absorption is known. This way, even with low photon statistics the coronal plasma of BD samples representing different evolutionary phases can be compared. From the median photon energy we deduce a temperature of G1569 Bab during quiescence of $\sim 0.3 \mathrm{keV}$, much lower than during the flare, and much lower than the temperature of HR 7329 B during quiescence, 
thus confirming the presumptions that (a) the coronal temperature decreases as BDs evolve, (b) this does not prevent the occurrence of large outbursts.

Our study has shown that despite the increasing data base on X-ray emission from BDs, the sensitivity of the existing observations is not always satisfactory. In future Chandra and $X M M$-Newton observations we hope to obtain better detection rates and to extend further the coverage of the age-massluminosity-temperature parameter space, to examine the influence of rotation, and to constrain the coronal temperatures near and beyond the substellar regime.

Acknowledgements. We would like to thank the referee K. Briggs for inspiring us to a critical scrutiny of the results, and for his care in reading the paper. B.S. acknowledges financial support from MIUR PRIN 2004-2006. Support for this work was provided by the National Aeronautics and Space Administration through Chandra Award Number 05200031 issued by the Chandra X-ray Observatory Center, which is operated by the Smithsonian Astrophysical Observatory for and on behalf of the National Aeronautics Space Administration under contract NAS8-03060.

\section{References}

Audard, M., Brown, A., Briggs, K. R., et al. 2005, ApJ, 625, L63

Baraffe, I., Chabrier, G., Allard, F., \& Hauschildt, P. H. 1998, A\&A, 337, 403

Baraffe, I., Chabrier, G., Allard, F., \& Hauschildt, P. H. 2002, A\&A, 382,563

Berger, E., Rutledge, R. E., Reid, I. N., et al. 2005, ApJ, 627, 960

Bouy, H. 2004, A\&A, 424, 619

Briggs, K. R., \& Pye, J. P. 2004, MNRAS, 353, 673

Chabrier, G., Baraffe, I., Allard, F., \& Hauschildt, P. 2000, ApJ, 542, 464

D’Antona, F., \& Mazzitelli, I. 1997, MemSAI, 68, 807

Favata, F., \& Micela, G. 2003, Space Sci. Rev., 108, 577

Feigelson, E. D., \& Nelson, P. I. 1985, ApJ, 293, 192

Feigelson, E. D., Broos, P., Gaffneu, J. A. III., et al. 2002, ApJ, 574, 258

Flaccomio, E., Micela, G., \& Sciortino, S. 2003, A\&A, 402, 277

Fleming, T. A., Giampapa, M. S., \& Schmitt, J. H. M. M. 2000, ApJ, 533, 372

Gaidos, E. J. 1998, PASP, 110, 1259

Gaidos, E. J., Henry, G. W., \& Henry, S. M. 2000, AJ, 120, 1006

Getman, K. V., Feigelson, E. D., Townsley, L., et al. 2002, ApJ, 575, 354

Getman, K. V., Flaccomio, E., Broos, E. S., et al. 2005, ApJS, 160, 319

Giampapa, M. S., Rosner, R., Kashyap, V., et al. 1996, ApJ, 463, 707

Gizis, J. E., \& Bharat, R. 2004, ApJ, 608, L113

Gizis, J. E., Monet, D. G., Reid, I. N., et al. 2000, AJ, 120, 1085

Goto, M., Kobayashi, N., Terada, H., et al. 2002, ApJ, 567, L59

Guenther, E. W., Neuhäuser, R., Huélamo, N., et al. 2001, A\&A, 365, 514

Hünsch, M., Schmitt, J. H. M. M., Sterzik, M. F., \& Voges, W. 1999, A\&AS, 135, 319

Jayawardhana, R., Mohanty, S., \& Basri, G. 2003, ApJ, 592, 282

Kraft, R. P., Burrows, D. N., \& Nousek, J. A. 1991, ApJ, 374, 344

Lachaume, R., Dominik, C., Lanz, T., \& Habing, H. J. 1999, A\&A, 348,897

Lane, B. F., Zapatero Osorio, M. R., Britton, M. C., Martin, E. L., \& Kulkarni, S. R. 2001, ApJ, 560, 390
Leggett, S. K., Allard, F., Geballe, T. R., Hauschildt, P. H., \& Schweitzer, A. 2001, ApJ, 548, 908

Lowrance, P. J., Schneider, G., Kirkpatrick, J. D., et al. 2000, ApJ, 541,390

Luhman, K. L. 1999, ApJ, 525, 466

Luhman, K. L. 2004, ApJ, 602, 816

Luhman, K. L., Stauffer, J. R., Muench, A. A., et al. 2003, ApJ, 593, 1093

MacGregor, K. B., \& Cassinelli, J. P. 2003, ApJ, 586, 480

Martín, E. L., \& Bouy, H. 2002, New Astron, 7, 595

McCaughrean, M. J., Close, L. M., Scholz, R.-D., et al. 2004, A\&A, 413, 1029

Mohanty, S., \& Basri, G. 2003, ApJ, 583, 451

Mohanty, S., Basri, G., Shu, F., Allard, F., \& Chabrier, G. 2002, ApJ, 571,469

Mohanty, S., Jayawardhana, R., \& Barrado y Navascués, D. 2003, ApJ, 593, L109

Mohanty, S., Jayawardhana, R., \& Basri, G. 2005, ApJ, 626, 498

Mokler, F., \& Stelzer, B. 2002, A\&A, 391, 1025

Muzerolle, J., Luhman, K. L., Briceño, C., Hartmann, L., \& Calvet, N. 2005, ApJ, 625, 906

Najita, J. R., Tiede, G. P., \& Carr, J. S. 2000, ApJ, 541, 977

Natta, A., Testi, L., Muzerolle, J., et al. 2004, A\&A, 424, 603

Neuhäuser, R., \& Comerón, F. 1998, Science, 282, 83

Neuhäuser, R., Briceño, C., Comerón, F., et al. 1999, A\&A, 343, 883

Perryman, M. A. C., Lindegren, L., Kovalevsky, J., et al. 1997, A\&A, 323, L49

Pizzolato, N., Maggio, A., Micela, G., et al. 2003, A\&A, 397, 147

Potter, D., Martín, E. L., Cushing, M. C., et al. 2002, ApJ, 567, L133

Preibisch, T., \& Zinnecker, H. 2002, AJ, 123, 1613

Preibisch, T., McCaughrean, M. J., Grosso, N., et al. 2005a, ApJS, 160, 582

Preibisch, T., Kim, Y.-C., Favata, F., et al. 2005b, ApJS, 160, 401

Raymond, J. C., \& Smith, B. W. 1977, ApJS, 35, 419

Ribas, I. 2003, A\&A, 400, 297

Rutledge, R. E., Basri, G., Martín, E. L., \& Bildsten, L. 2000, ApJ, 538, L141

Ryter, C. E. 1996, Ap\&SS, 236, 285

Schmitt, J. H. M. M., \& Liefke, C. 2002, A\&A, 382, L9

Scholz, A., Jayawardhana, R., \& Brandeker, A. 2005, ApJ, 629, L41

Scholz, R.-D., Lodieu, N., Ibata, R., et al. 2004, MNRAS, 347, 685

Silvestri, N. M., Oswalt, T. D., Wood, M. A., et al. 2001, AJ, 121, 503

Smith, R. K., Brickhouse, N. S., Liedahl, D. A., \& Raymond, J. C. 2001, ApJ, 556, L91

Stelzer, B. 2004, ApJ, 615, L153

Stelzer, B., \& Neuhäuser, R. 2000, A\&A, 361, 581

Stelzer, B., \& Neuhäuser, R. 2001, A\&A, 377, 538

Stelzer, B., \& Neuhäuser, R. 2002, in Proc. of IAU Symp. 211, Brown Dwarfs, May 21-24, 2002, Big Island, Hawaii, USA, ed. E. L. Martín, 2003, San Francisco: Astronomical Society of the Pacific, 443

Stelzer, B., Micela, G., \& Neuhäuser, R. 2004, A\&A, 423, 1029

Telleschi, A., Güdel, M., Briggs, K., et al. 2005, ApJ, 622, 653

Tinney, C. G. 1998, MNRAS, 296, L42

Tsuboi, Y., Maeda, Y., Feigelson, E. D., et al. 2003, ApJ, 587, L51

Wolk, S. J., Harnden, F. R. Jr., Flaccomio, E., et al. 2005, ApJS, 160, 423

Zapatero Osorio, M. R., Lane, B. L., Pavlenko, Y., et al. 2004, ApJ, 615,968

Zuckerman, B., \& Webb, R. A. 2000, ApJ, 535, 959

Zuckerman, B., Song, I., Bessell, M. S., \& Webb, R. A. 2001a, ApJ, 562, L87

Zuckerman, B., Song, I., \& Webb, R. A. 2001b, ApJ, 559, 388 